\title{
STATUS OF THE MAX IV LIGHT SOURCE PROJECT
}

\author{
Mikael Eriksson*, Bengt Anderberg, Ingvar Blomqvist, Mathias Brandin, Magnus Berglund, Tue \\ Hansen, Dionis Kumbaro, Lars-Johan Lindgren, Lars Malmgren, Hamed Tarawneh, Sara Thorin, \\ Magnus Sjöström, Håkan Svensson, Erik Wallén, and Sverker Werin. \\ MAX-lab, Box 118, 22100 Lund, Sweden
}

\section{Abstract}

The present development of the accelerator part of the MAX IV synchrotron radiation project is presented. The main features of the $3 \mathrm{GeV}$ injector linac and the two storage rings operated at different electron energies to cover a broad spectral range of high brilliance undulator radiation are described in some detail. A third ring, the existing MAX III ring is planned to be transferred to the new facility. The preparation of the injector linac to serve as a Free Electron Laser source and the major subsystems of the facility are also presented.

\section{INTRODUCTION}

A fundamental challenge for the design of the proposed MAX IV facility is that the scientific demands call for a facility which will deliver spontaneous as well as coherent radiation of very high quality over a broad spectral range, from IR radiation, VUV radiation and soft $\mathrm{X}$-rays to hard X-rays. We have performed a global optimization of the design based on these broad demands. This has resulted in a construction where two MAX IV storage rings will provide spontaneous radiation of high brilliance. The two rings, each with 12-fold symmetry, will be operated at around $1.5 \mathrm{GeV}$ and at $3.0 \mathrm{GeV}$, respectively. A new construction of the magnetic lattices has allowed us to place the two MAX IV rings on top of each other. The prime sources for synchrotron radiation will consist of undulators and wigglers, which will span the photon energy range from below hundred $\mathrm{eV}$ up to several tens of $\mathrm{keV}$. The plan is also to complement the two new rings by the $0.7 \mathrm{GeV}$ MAX III ring, which will be relocated from the present laboratory. All rings will be operated in top-up mode. The MAX III ring will be the prime source for IR and UV radiation and extends the photon energy range for undulator radiation down to a few eV. The Linac will also be used to generate short spontaneous X-ray pulses and coherent radiation in a second phase. The MAX IV storage rings will provide electron beams with extremely small emittance. The construction of a facility with two rings, one for higher and one for lower energies has allowed an optimization with very few compromises. For the high energy ring an energy of $3.0 \mathrm{GeV}$ has been found to be (cost) optimal. The exact electron energy for the other ring has not yet been decided. However, the energy will be rather close to 1.5 GeV and we will in the following refer to this as the $1.5 \mathrm{GeV}$ ring. The MAX IV project is not yet financed but the fund raising process of the project has started.

\section{LAYOUT OF THE MAX IV FACILITY}

The MAX IV facility layout is shown in Fig. 1. The $1.5 \mathrm{GeV}$ storage ring is placed on top of the $3.0 \mathrm{GeV}$ ring. The two rings are rotated $15^{\circ}$ relative to each other. The beamlines from the straight sections will thus be spread out evenly on the hall floor. The MAX III storage ring is located in the area into which the two injection straight sections are pointing. This area can anyhow not be used for beamlines on the MAX IV rings but it is large enough

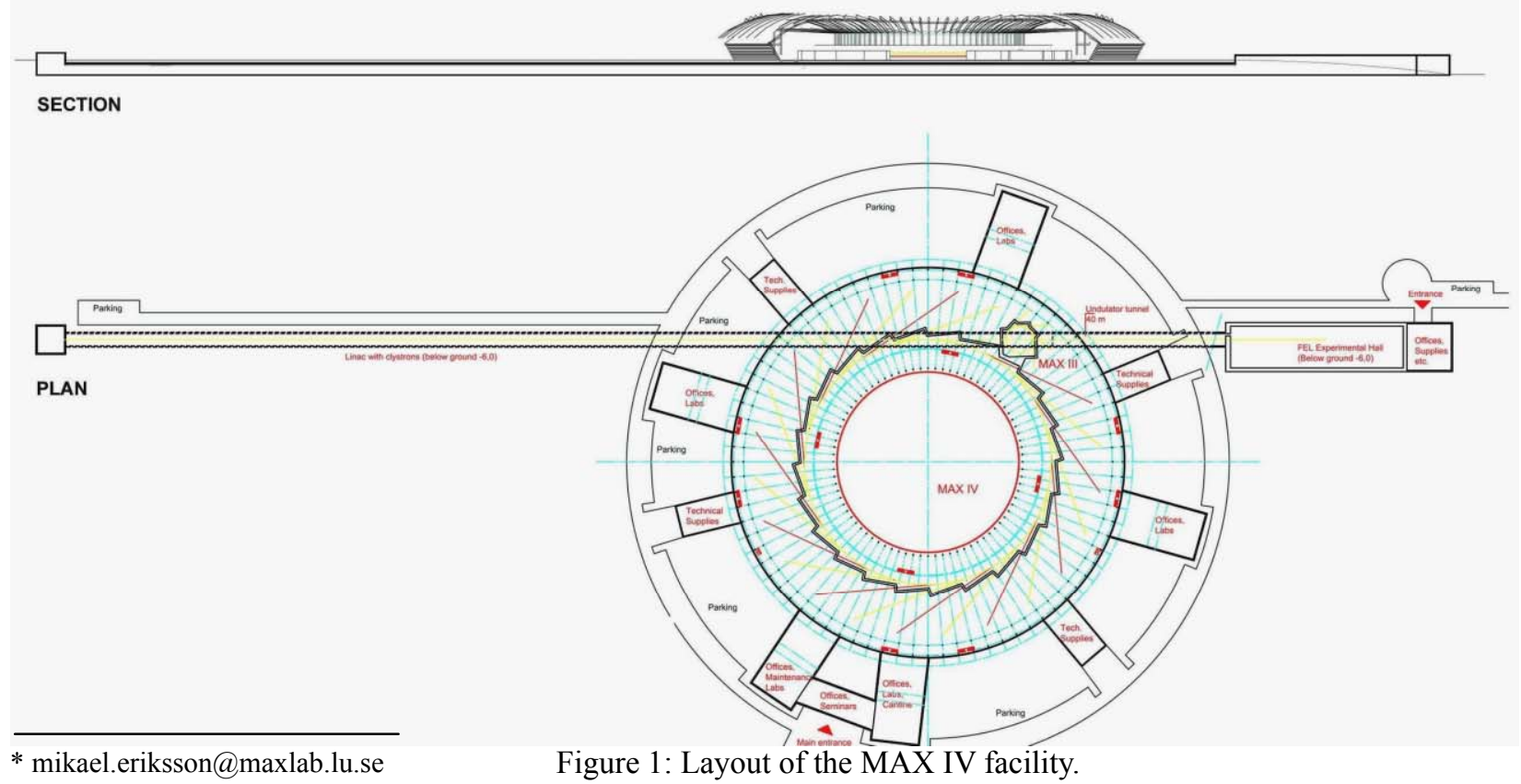


for at least four insertion device beamlines on MAX III. IR beamlines can be placed on the roof of the radiation shield.

The $3 \mathrm{GeV}$ injector Linac is housed in a $300 \mathrm{~m}$ long tunnel, 5-7 m below the floor level of the MAX IV rings. The beam is brought up to the storage rings via an achromatic transport line. A Lambertson injection septum in each ring will deflect the beam into the respective storage ring. The undulators for the coherent generation and the undulators and/or wigglers for short pulse generation are placed close to the Linac axis and below the ground level. The experimental hall for the short pulse applications will be situated right after the undulator/wiggler hall. This layout allows for future expansions of the activity. The Linac tunnel as well as the short pulse experimental hall can be expanded without interfering with the ongoing activity at the storage rings.

\section{STORAGE RINGS}

The magnet lattices of the $1.5 \mathrm{GeV}$ and $3.0 \mathrm{GeV}$ storage rings are identical. The parameters of the rings are summarized in Table 1 . The $0.7 \mathrm{GeV}$ MAX III ring has been described elsewhere [1].

Many of the ideas for the MAX IV storage rings have been inspired by the design of high energy physics damping rings $[2,3]$. These rings are characterized by a very low electron beam emittance, high bunch currents and also by small transverse dimensions regarding apertures and magnet elements.

The MAX IV design focuses on both the soft and hard $\mathrm{X}$-ray regimes. To achieve this in one single ring would require a $3.0 \mathrm{GeV}$ ring equipped with a number of very long straight sections. An alternative approach, made possible by the compact MAX IV design, is to add a second ring at $1.5 \mathrm{GeV}$ energy on top of the $3.0 \mathrm{GeV}$ ring. The low photon energy beamlines are then placed in a

\begin{tabular}{|c|c|c|c|}
\hline Ring & $0.7 \mathrm{GeV}$ & $1.5 \mathrm{GeV}$ & $3.0 \mathrm{GeV}$ \\
\hline Circumference $[\mathrm{m}]$ & 36 & 287.2 & 287.2 \\
\hline Electron energy [GeV] & 0.7 & 1.5 & 3 \\
\hline Circulating current $[\mathrm{A}]$ & 0.5 & 0.5 & 0.5 \\
\hline Energy acceptance $[\%]$ & 2 & 3 & 3 \\
\hline Horizontal emittance [nm & 14 & 0.34 & $0.8 *$ \\
\hline Momentum compaction & 0.035 & $7.45 \times 10^{-4}$ & $7.45 \times 10^{-4}$ \\
\hline Betatron tunes Qx/Qy & $3.7 / 2.7$ & $26.59 / 9.184$ & $26.59 / 9.184$ \\
\hline Coupling & 0.1 & 0.1 & 0.01 \\
\hline $\mathrm{Nr}$ of straight sections & 8 & 12 & 12 \\
\hline Straight section length [m] & 3 & 4.6 & 4.6 \\
\hline $\mathrm{RF}[\mathrm{MHz}]$ & 100 & 100 & 100 \\
\hline Energy loss/turn [keV] & 7 & 42 & 712 \\
\hline Power consump. [MW] & 0.2 & 0.5 & 1 \\
\hline
\end{tabular}

* Incuding superconducting wigglers

ring of lower electron energy. Long undulator periods and high K-values can thus be avoided.

To achieve a small electron beam emittance a large number of focusing elements is needed. For a given ring size one can increase the number of magnet cells by making the cells smaller. Shorter magnet elements calls for stronger focusing gradients to keep the electron optics optimised. The strong gradients needed for the MAX IV rings are achieved by a reduction of the transverse dimensions of the magnet elements which results in a small bore radius. The general trend in light source design is that the magnet apertures are decreased. Small gap insertion devices will anyhow restrict the aperture of the rings. Whenever it is advantageous, different multipole components are integrated into the same magnet elements. The dipole magnets are equipped with strong magnet gradients and the focusing quadrupoles have sextupole and octupole components.

A Multiple Bend Achromat structure with highly integrated magnet functions has thus been chosen for the MAX IV lattice. Each storage ring consists of 12 supercells, a supercell is defined as the magnet structure between the long straight sections housing the IDs. Each supercell is built up by 5 unit cells, a unit cell consists of a dipole magnet and two flanking half quadrupole magnets and the sextupole magnets needed for chromaticity correction. Two matching sections are added to optimise the machine functions in the 12 straight sections for the insertion devices. The vertical focusing is taken care of by the gradients of the dipole magnets [4]. In the matching sections, soft end dipole magnets are introduced to reduce the synchrotron radiation power hitting the downstream straight section. This will simplify the introduction of superconducting insertion devices with small gaps. The chromaticity correction is taken care of by the integrated sextupole component in the quadrupole magnets and by discrete sextupoles flanking the dipole magnets. The lattice functions are seen in Fig. 2.

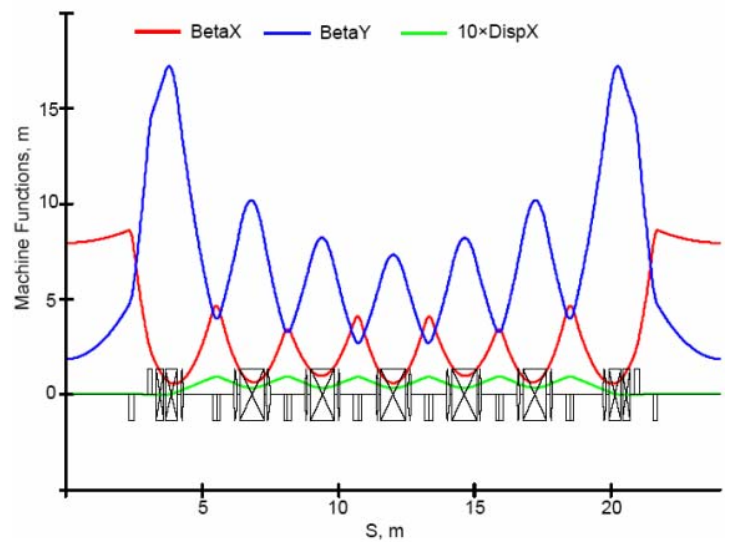

Figure 2: Machine functions of the 1.5 and $3.0 \mathrm{GeV}$ rings.

The magnets will be machined out of solid iron blocks resulting in an integrated girder-magnet design. A $24 \mathrm{~mm}$ gap in the bending and quadrupole magnets is foreseen since the small gap insertion devices anyhow will define the ring admittance. The small aperture allows for the introduction of strong multipole fields as mentioned above.

The Radio Frequency (RF) system will probably be a $100 \mathrm{MHz}$ system. The cavities will be similar to the three $100 \mathrm{MHz}$ cavities currently in operation at the MAX II 
storage ring and the cavity placed in the MAX III ring [5]. Landau cavities will be used to decrease the electron density and thus increase the Touschek beam lifetime and also decrease the heat load on the insertion devices from the resistive wall effect.

\section{INSERTION DEVICES}

The most challenging insertion devices for the MAX IV storage rings are the insertion devices for beamlines on the $3.0 \mathrm{GeV}$ storage ring requiring high brilliance radiation in the hard X-ray regime. These undulators will by necessity be in-vacuum insertion devices since a small magnetic gap is needed in order to obtain a high undulator peak field in combination with a short period length.

Superconducting undulators give a higher peak field than undulators based on permanent magnet technology. Superconducting undulators, however, do not yet represent a mature technology and when estimating the brilliance of the $3.0 \mathrm{GeV}$ MAX IV ring it is also necessary to include the present technology, which is invacuum hybrid type undulators with permanent magnets. The performance of the permanent magnet material may also be increased by lowering the temperature of the permanent magnet material to $100-150 \mathrm{~K}$ [6].

Two superconducting wigglers will be installed in the $3.0 \mathrm{GeV}$ ring. The function of the superconducting wigglers is twofold, they are sources of synchrotron radiation for experimental stations but they are also working as damping wigglers, lowering the emittance of the storage ring.

The straight sections of the MAX IV storage rings are $4.6 \mathrm{~m}$ long and the vertical beam stay clear aperture is 4 $\mathrm{mm}$ in the $3.0 \mathrm{GeV}$ ring and $6 \mathrm{~mm}$ in the $1.5 \mathrm{GeV}$ ring. The expected brilliance at the $3.0 \mathrm{GeV}$ ring for a superconducting undulator with $14 \mathrm{~mm}$ period length (SCU 14.0), a cryogenically cooled hybrid undulator with $17.5 \mathrm{~mm}$ period length (CPU 17.5), and an in in-vacuum

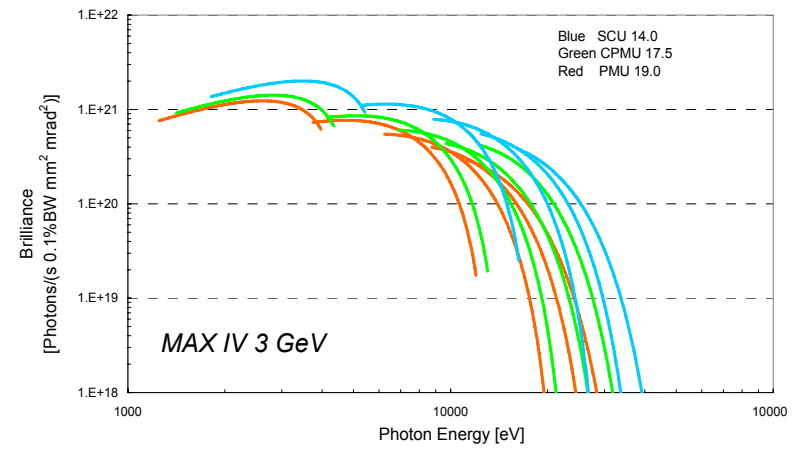

Figure 3: Brilliance at the $3.0 \mathrm{GeV}$ ring for the harmonics 1, 3,5 , and 7 of undulators with a $\mathrm{K}$-value in the range 0.5-2.2.

undulator with $19 \mathrm{~mm}$ period length (PMU 19.0) are shown in Fig. 3.

The small vertical dimension of the straight section vacuum tube makes the MAX IV storage ring ideal for the installation of out-of-vacuum devices, such as conventional planar undulators or elliptically polarizing undulators.

\section{INJECTOR AND SHORT PULSE GENERATION}

The full energy injector is an S-band linac and the basic parameters for the injector are given in Table 2. The S-band structures are driven by SLED systems [7] and high power klystrons. The Linac will be able to operate in either a high energy mode or high repetition mode. The SLED cavities will be used in the high energy mode. This mode will be used for injection into the storage rings. The high energy mode can also be used for the production of short pulses of X-rays or for FEL operation. In the high repetition rate mode, the SLED cavities are shortcircuited and the klystron power is fed directly into the Linac. This mode is motivated by those VUV FEL experiments that require a higher repetition rate. The two main modes of operation of the Linac system require two or more different Linac pre-injector systems. One system will be used for storage ring injection with top-up capabilities and the others will be used for FEL/short pulse operation.

The top-up injection schemes could be realised in several ways. One possible scheme is to allow for a current variation of $1 \%$ by injection every few minutes, depending on the actual beam life-time. Each injection will last for a few seconds at most. In the time between, $95 \%$ of the total time, the linac can be used for the production of pulsed light. Short ps or fs pulses in the Xray spectral region can be produced by feeding an undulator with short Linac pulses. The Linac is thus prepared for the operation as an electron source for coherent radiators. This will be the second phase of the construction and different types of FELs are presently being considered

Table 2: Linac injector

\begin{tabular}{lll}
\hline & \multicolumn{2}{l}{ Without SLED With SLED } \\
\hline Endpoint energy $[\mathrm{GeV}]$ & 2 & 3.5 \\
Length $[\mathrm{m}]$ & 300 & 300 \\
Rep rate $[\mathrm{Hz}]$ & 500 & 100 \\
Nr of klystron modules & $15-20$ & $15-20$ \\
Max power consumption $[\mathrm{MW}]$ & 2.5 & 1.5 \\
\hline
\end{tabular}

\section{REFERENCES}

[1] G. LeBlanc, Å. Andersson, M. Eriksson, M. Georgsson, L-J. Lindgren, and S. Werin, Proc. of EPAC 2000, Vienna, Austria.

[2] M. Korostelev and F. Zimmermann, Proceedings of EPAC 2002, Paris, France.

[3] A. Wolski and J. N. Corlett, Proceedings of PAC 2001.

[4] H. Tarawneh, M. Eriksson, L-J. Lindgren and B. Anderberg, Nucl. Instr. and Meth. A508, 480-486 (2003).

[5] Å. Andersson, et al., Proceedings of EPAC 2002, Paris, France.

[6] T. Hara, et. al., Phys. Rev. ST-AB, V 7, 050702 (2004).

[7] Z.D. Farkas et al., SLAC-PUB-1453, June 1974. 